\title{
Detection Thresholds of Macaque Otolith Afferents
}

\author{
Xiong-jie Yu, ${ }^{1}$ J. David Dickman, ${ }^{2}$ and Dora E. Angelaki ${ }^{1,2}$ \\ ${ }^{1}$ Department of Anatomy and Neurobiology, Washington University School of Medicine, St. Louis, Missouri 63110, and ${ }^{2}$ Department of Neuroscience, \\ Baylor College of Medicine, Houston, Texas 77030
}

The vestibular system is our sixth sense and is important for spatial perception functions, yet the sensory detection and discrimination properties of vestibular neurons remain relatively unexplored. Here we have used signal detection theory to measure detection thresholds of otolith afferents using $1 \mathrm{~Hz}$ linear accelerations delivered along three cardinal axes. Direction detection thresholds were measured by comparing mean firing rates centered on response peak and trough (full-cycle thresholds) or by comparing peak/trough firing rates with spontaneous activity (half-cycle thresholds). Thresholds were similar for utricular and saccular afferents, as well as for lateral, fore/aft, and vertical motion directions. When computed along the preferred direction, full-cycle direction detection thresholds were 7.54 and 3.01 $\mathrm{cm} / \mathrm{s}^{2}$ for regular and irregular firing otolith afferents, respectively. Half-cycle thresholds were approximately double, with excitatory thresholds being half as large as inhibitory thresholds. The variability in threshold among afferents was directly related to neuronal gain and did not depend on spike count variance. The exact threshold values depended on both the time window used for spike count analysis and the filtering method used to calculate mean firing rate, although differences between regular and irregular afferent thresholds were independent of analysis parameters. The fact that minimum thresholds measured in macaque otolith afferents are of the same order of magnitude as human behavioral thresholds suggests that the vestibular periphery might determine the limit on our ability to detect or discriminate small differences in head movement, with little noise added during downstream processing.

\section{Introduction}

For decades, the neural processes underlying vestibular function have been primarily studied in relationship to reflexes, and these efforts were dominated by the use of linear systems analysis tools. In contrast, relatively little has been done to understand the neural basis of perception related to self-motion and spatial orientation around psychophysical threshold, in which linear systems analysis tools are rather limited (for review, see MacNeilage et al., 2008). For otolith system function, in particular, we have a limited understanding of the neural mechanisms underlying sensory psychophysics (De Vrijer et al., 2008; Zupan and Merfeld 2008; MacNeilage et al., 2010a,b). More recently, questions related to vestibular neuronal thresholds have started to be explored in central neurons (Gu et al., 2007; Liu et al., 2010). However, neither central responses nor perception can be well understood and modeled without a quantitative characterization of the sensory detection and discrimination properties of otolith afferents. The few studies using approaches beyond classical linear control system tools in the vestibular periphery have all focused on semicircular canals (Paulin and Hoffman 1999, 2001; Sadeghi et al., 2007). In contrast, otolith afferents have only been quantified using linear systems analyses (gerbil: Dickman et al., 1991; Purcell

Received March 4, 2012; revised April 8, 2012; accepted April 28, 2012.

Author contributions: D.E.A. designed research; X.-J.Y. and J.D.D. performed research; X.-J.Y. analyzed data; J.D.D. and D.E.A. wrote the paper.

The work was supported by National Institutes of Health Grant R01 DC04260. We thank Paul MacNeilage for comments on this manuscript.

Correspondence should be addressed to Dr. Dora Angelaki, Department of Neuroscience, Room S740, MS BCM295, Baylor College of Medicine, One Baylor Plaza, Houston, TX 77030. E-mail: angelaki@bcm.edu.

DOI:10.1523/JNEUROSCI.1067-12.2012

Copyright $\odot 2012$ the authors $\quad 0270-6474 / 12 / 328306-11 \$ 15.00 / 0$ et al., 2003; cat: Loe et al., 1973; Anderson et al., 1978; squirrel monkey: Fernández et al., 1972; Fernández and Goldberg 1976a-c; pigeon: Si et al., 1997; macaque monkey: Angelaki and Dickman 2000; Angelaki et al., 2004; Jamali et al., 2009; chinchilla: Goldberg et al., 1990a,b).

Using such linear control system approaches, otolith afferents have been characterized in terms of preferred direction (PD) in three dimensions (3D) (Fernández and Goldberg 1976a), response dynamics (Fernández and Goldberg 1976c; Goldberg et al., 1990a; Si et al., 1997; Angelaki and Dickman 2000; Purcell et al., 2003), and coding of net gravito-inertial acceleration (Angelaki and Dickman 2000; Angelaki et al., 2004). Here we explore for the first time direction thresholds of linear acceleration in the macaque peripheral vestibular system. Direction detection thresholds, defined as the peak acceleration/deceleration that allows direction discrimination with a probability of $84 \%$ correct, were measured by comparing mean firing rates centered on response peak and trough (full-cycle detection thresholds). The goal of this analysis was to evaluate how reliably each afferent fiber can discriminate peak from trough (e.g., leftward from rightward) linear acceleration, a property that is functionally relevant for naturalistic, commonly experienced, transient (start/ stop) movements. In addition, we also compared peak/trough firing rates with spontaneous activity (half-cycle detection thresholds). In the real world, such a stimulus would correspond to either the start or stop of constant velocity translation. Characterization of linear acceleration thresholds in the vestibular periphery is fundamental for understanding information processing within the central vestibular system and how neuronal sensitivity can constrain behavioral sensitivity and perceptual thresholds during linear acceleration (Benson et al., 1986; Zupan and Merfeld 2008; MacNeilage et al., 2010a,b). 
Parts of this work have been published previously in abstract form (Yu et al., 2011).

\section{Materials and Methods}

Experiments were conducted using a 6-degree-of-freedom motion platform (Moog 6DOF2000E). Data were collected in two male rhesus monkeys (Macaca mulatta) which were chronically implanted with a plastic head-restraint ring and a guide tube platform, as described in detail previously (Meng et al., 2005; Gu et al., 2006; Yakusheva et al., 2008). All surgical procedures were performed under sterile conditions in accordance with institutional and National Institutes of Health guidelines.

We recorded extracellular neural activity of single otolith afferents using high-impedance, epoxy-coated tungsten microelectrodes (FHC). Electrodes were inserted into 26-gauge transdural guide tubes and advanced by a remote-controlled microdrive (FHC). The vestibular nerve was isolated beneath the auditory meatus as it entered the brain (Haque et al., 2004). Raw neural activity was amplified, filtered $(0.1 \mathrm{~Hz}$ to 10 $\mathrm{kHz}$ ), and passed through a dual time-amplitude window discriminator (BAK Electronics). The spike train of each vestibular afferent was analyzed offline. Single units were identified based on waveform shape, latency, and amplitude. Only well-isolated neurons were included for analyses.

Experimental protocol. Each otolith afferent was first tested during 0.5 $\mathrm{Hz}$ oscillations along the three cardinal axes, i.e., lateral [LR; peak, $0.1 \mathrm{G}$, where $\mathrm{G}=981 \mathrm{~cm} / \mathrm{s}^{2}$ ], naso-occipital (NO; peak, $0.1 \mathrm{G}$ ). and dorsoventral (DV; peak, $0.07 \mathrm{G}$ ). These responses were used to characterize the PD of the cell in 3D. In addition, the spontaneous activity was also recorded for $20 \mathrm{~s}$ with the animal upright and stationary. After completion of this "classification" battery of tests, the main experimental protocol consisted of $1 \mathrm{~Hz}$ sinusoidal oscillations at different peak linear acceleration amplitudes $(0.005,0.01,0.02,0.04,0.08$, and $0.16 \mathrm{G})$. Most cells were tested with $>40$ cycles at each linear acceleration amplitude. For a few cells in initial experiments, this protocol was delivered first along the cardinal direction (i.e., LR, NO, or DV) that the cell responded the most (based on the classification $0.5 \mathrm{~Hz}$ sinusoids). For most of the neurons, however, this $1 \mathrm{~Hz}$ variable-stimulus-amplitude protocol was first delivered along the LR direction, then the NO direction, and last (if isolation was maintained) along the DV direction. Only for some neurons (17 of 49), data from all three directions were collected. By comparing responses to different linear acceleration magnitudes, we could measure the smallest stimulus amplitude that an ideal observer could detect based on the firing rate of the particular neuron. All data were collected in complete darkness, and animals were always sitting upright relative to the motion platform and gravity.

Data analysis. Sinusoidal responses during the $0.5 \mathrm{~Hz}$ linear accelerations along the cardinal directions (classification protocol) were first quantified using instantaneous firing rate (IFR), computed as the inverse of the interspike interval (ISI) (see Fig. $1 \mathrm{~A}$ ). We then stacked responses to repeated stimulus cycles into a single IFR cycle. Gain and phase were then calculated by fitting both neural response (clipped off at zero response) and stimulus with a sine function (first and second harmonics and direct current offset) using a nonlinear least-squares algorithm (LevenbergMarquardt methods). Response amplitude was then measured to be half the peak-to-trough first harmonic modulation of the sinusoidal fit. Neuronal gain for translation was computed as the ratio of response modulation amplitude over the stimulus (in units of spikes/s/G). Phase was expressed relative to peak linear acceleration. These gain values along the three cardinal directions were subsequently used to compute the threedimensional PD and its response gain based on a cosine model for response tuning (Fernández and Goldberg, 1976b). The response phase used in summary plots (see Fig. 2) is the phase along the cardinal direction that gave the largest response. Note that, given the sinusoidal stimuli, here we define response peak as the "excitatory" and response trough as the "inhibitory" component of the modulation of the cell around spontaneous activity.

For the threshold calculations, we converted the firing rate during the variable-magnitude $1 \mathrm{~Hz}$ sinusoidal stimulation into a continuous function using either (1) a low-pass filter with a Kaiser window and cutoff frequency greater than the stimulus frequency by $0.1 \mathrm{~Hz}$ (Sadeghi et al.,
2007; Jamali et al., 2009) (see Fig. $1 B$, gray lines) or (2) a spike density function with a variable width, $\sigma$. The first method was used for the main threshold analyses (see Figs. 3-6), whereas the latter method was used to illustrate that the exact neuronal threshold values measured depended on the analysis method used (see Fig. 9). For the same reason, we also varied the temporal window during which mean firing rates were computed in Figure 9 (the main analyses used a temporal window of $100 \mathrm{~ms}$ ).

Our first goal was to measure direction detection thresholds, i.e., the sensitivity of neurons to changes in the direction of linear acceleration for each cardinal direction. To do this, we needed to compare distributions of firing rates (across multiple cycles) for each trough and peak of response modulation along each cardinal direction of translation. These distributions were then subjected to receiver operating characteristic (ROC) analysis to compute discriminability (Gu et al., 2007, 2008). To measure the timing of response peak and trough (because response phase is generally non-zero), we shifted the time of each zero-crossing of the stimulus according to the phase of the measured neural response, using the phase estimated from the sinusoidal fit. Thus, for each cycle of motion, we computed two firing rates, each centered on trough and peak (see bars in Fig. $3 A$ ), corresponding to each half-cycle of the neural response. Translation directions for these two half-cycles corresponded to opposite stimulus directions, such that each stimulus cycle yielded two firing rates representing headings that differed by $180^{\circ}$.

Having transformed neural responses into firing rates for each direction of motion, we then used ROC analysis (Britten et al., 1992; Gu et al., 2007 ) to compute a neuronal direction detection threshold that quantifies the ability of an ideal observer to discriminate between two opposite directions of motion based on the firing rates of a particular cell (fullcycle detection thresholds). This was done by constructing and comparing firing rate distributions for pairs of directions (i.e., left and right during LR motion) for each stimulus amplitude (e.g., 0.005, 0.02, 0.04, $0.16 \mathrm{G}$, etc.) (see Fig. $3 B$ ). For each pair of symmetrically spaced "comparison" directions, distributions of firing rates were compiled and an ROC metric (Green and Swets, 1966) was computed. The proportion of "one choice" (i.e., rightward during LR motion), taken as the area under the corresponding ROC curve, was then plotted against stimulus amplitude, yielding a neurometric function. The neurometric function was fit by a cumulative Gaussian function (see Fig. 3D), and neuronal threshold was defined as the SD of the Gaussian, which corresponded to $84 \%$ correct performance.

In addition, linear acceleration half-cycle detection thresholds were computed in a similar way but, rather than comparing left and right distributions with each other, we instead compared each of the peaks (excitatory detection threshold) and troughs (inhibitory detection threshold) with a similar time course of spontaneous activity $(100 \mathrm{~ms}$ intervals sampled randomly from the $20 \mathrm{~s}$ spontaneous activity trial; see above, Experimental protocol). Whereas the goal of the previous analysis was to evaluate how reliably the neuron can discriminate peak from trough activation (e.g., left from right linear acceleration), the goal of this analysis was to characterize how reliably the neuron can discriminate peak or trough activation from spontaneous activity. Theoretically, if (1) mean peak and trough responses are symmetrically spaced around spontaneous activity and (2) the spike count variability of peak, trough, and spontaneous activity are all equal, then half-cycle detection thresholds should be twice as large as full-cycle detection thresholds (because differences in firing rate distribution means for half-cycle analysis would be half those for full-cycle detection threshold analysis).

To quantify the linearity of magnitude tuning curves, we fitted both linear $(y=b x+a)$ and quadratic $\left(y=c x^{2}+b x+a\right.$, where $y$ is peak/ trough firing rate, and $x$ is peak/trough linear acceleration amplitude) equations to the magnitude tuning curves for each stimulus direction tested (see Fig. 7). The relative quality of the fits was evaluated using the Akaike information criterion (AIC) (Akaike, 1992):

$$
\mathrm{AIC}=N \times \ln \left(\frac{\mathrm{SS}}{N}\right)+2 \mathrm{~K}
$$

where $N$ is the number of data points, $K$ is the number of model parameters, and SS is the sum squared error. The AIC depends on both the 
goodness-of-fit and the number of independent parameters included in each model. The best model based on this criterion is the one with the lowest AIC value. Thus, for cells with a lower quadratic AIC (compared with the linear AIC), the difference in sum squared error between the linear and quadratic models is greater than that expected based on the difference in the number of parameters, implying that the model with more parameters (quadratic) provides a better characterization of the data.

Analysis of background activity. To classify otolith afferents based on discharge regularity, the distribution of ISIs recorded during spontaneous activity was used to compute the coefficient of variation $(\mathrm{CV}): \mathrm{CV}=\sigma_{\mathrm{ISI}} / \mu_{\mathrm{ISI}}$, where $\sigma_{\text {ISI }}$ and $\mu_{\text {ISI }}$ were the mean and SD of the ISI distribution. Because $\mathrm{CV}$ varies with the mean ISI, we used a normalized measure, $\mathrm{CV}^{\star}$, to classify otolith afferents (Goldberg et al., 1990a,b; Jamali et al., 2009). Neurons with $\mathrm{CV}^{\star}$ $<0.10$ were classified as regular, whereas those with a $\mathrm{CV}^{*}>0.10$ were classified as irregular.

\section{Results}

Basic response characteristics

We recorded from 49 otolith afferents in two rhesus macaques ( 21 cells in monkey $\mathrm{K}$ and 28 cells in monkey $\mathrm{H}$ ). As illustrated by plotting IFR for one example cell in Figure $1 A$, each fiber was first characterized in terms of its spontaneous activity and its modulation during $0.5 \mathrm{~Hz}$ linear acceleration oscillations along each of the cardinal axes, LR (top), NO (middle), and DV (bottom). From these responses, three-dimensional PD, gain, and phase were computed using a cosine-tuning model. Basic response characteristics are summarized in Figure 2. Response gains of otolith afferents showed a strong dependence on discharge regularity, quantified by the normalized $\mathrm{CV}^{\star}$ (see Materials and Methods). As also shown in previous studies (Fernández and Goldberg, 1976a-c; Goldberg et al., 1990a,b; Jamali et al., 2009), the more irregular the firing rate of the fiber, the higher its gain (Fig. $2 A$ ) and the larger the phase lead relative to linear acceleration (Fig. $2 B$ ). Both of these relationships were significant (gain vs $\mathrm{CV}^{\star}, r=0.86, p<0.001$; phase vs $\mathrm{CV}^{\star}, r=0.76, p<0.001$, type II regression). Gains at $0.5 \mathrm{~Hz}$ varied from $<30$ spikes/s/G to $>300$ spikes/ $\mathrm{s} / \mathrm{G}$, and this range is consistent with previous studies (Goldberg et al., 1990a,b; Jamali et al., 2009).

In our sample, there were 36 regular otolith afferents $\left(\mathrm{CV}^{\star}<\right.$ $0.1)$ and 13 irregular otolith afferents $\left(\mathrm{CV}^{\star}>0.1\right)$. The substantially higher proportion of regular otolith afferents is consistent with previous reports (Fernández et al., 1972; Fernández and Goldberg, 1976a; Goldberg et al., 1990a,b). Linear acceleration gains $(0.5 \mathrm{~Hz})$ averaged $41.49 \pm 1.72$ and $219.65 \pm 1.36$ spikes/s/G for regular and irregular otolith afferents, respec-
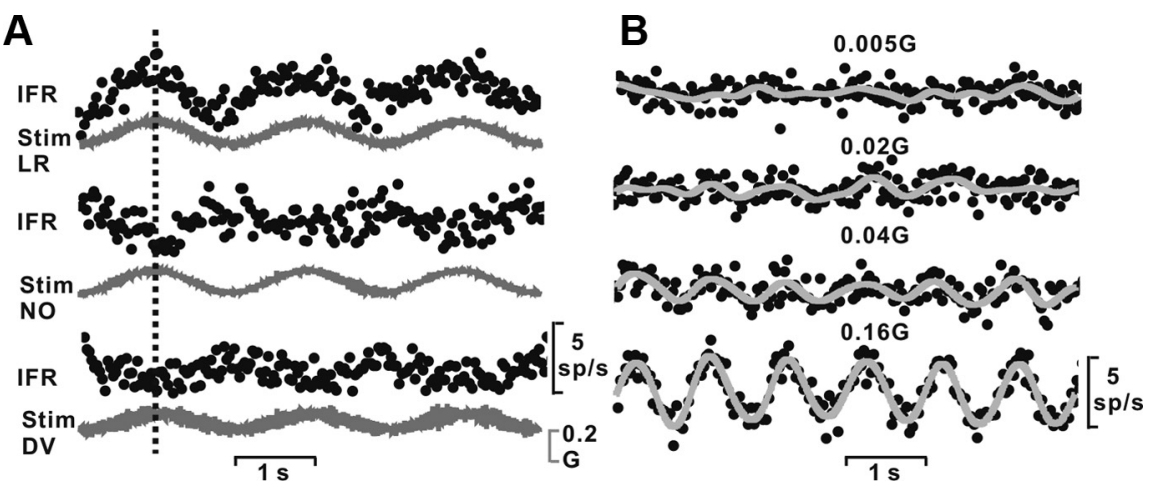

Figure 1. Example otolith afferent response, shown as IFR. $A$, Modulation during three cycles of sinusoidal linear acceleration along the LR (top), N0 (middle), and DV (bottom) axes. Stimuli (0.5 Hz) are shown below the IFR (LR, N0, 0.1 G; DV, 0.07 G). B, Experimental protocol used to measure detection thresholds. From top to bottom, Response to different magnitudes of $1 \mathrm{~Hz} L \mathrm{LR}$ linear acceleration. Gray lines illustrate Kaiser filter (see Materials and Methods) superimposed on top of the IFR.
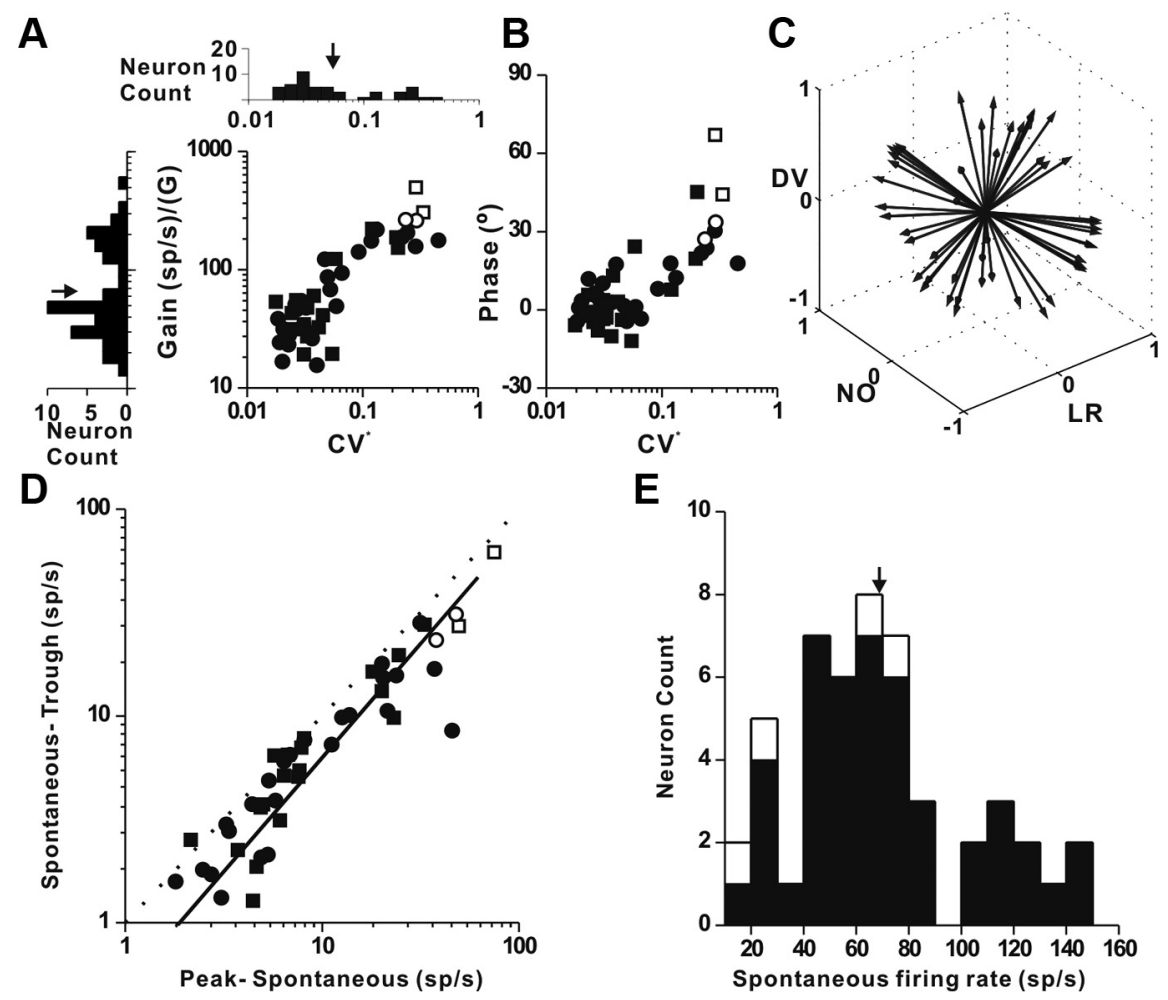

Figure 2. Summary of basic response properties of macaque otolith afferents $(n=49)$. $\boldsymbol{A}$, Response gain (along the 3-dimensional PD) as a function of $\mathrm{CV}^{*}$. Marginal histograms show gain and $\mathrm{CV}^{*}$ distributions (arrows illustrate geometric means). $\boldsymbol{B}$, Response phase (measured during linear acceleration along the cardinal axis that elicited the largest response for each cell) as a function of $\mathrm{CV}^{*}$. C, PD vector distribution in three-dimensional space (all magnitudes have been normalized to a gain of 1). $\boldsymbol{D}$, Scatter plot of the difference between response peak and spontaneous activity versus the difference between spontaneous activity and response trough (averaged over $100 \mathrm{~ms}$; see Materials and Methods). Data are only shown for the largest response amplitude (1 data point per cell). Dotted line, Unity slope; solid line, type II linear regression. $\boldsymbol{E}$, Distribution of spontaneous firing rates. Open symbols/bars illustrate neurons (all irregular fibers) with silencing of activity during the inhibitory response. Different symbols in $\boldsymbol{A}, \boldsymbol{B}$, and $\boldsymbol{D}$ are used for different animals (squares, monkey $\mathrm{K}$; circles, monkey $\mathrm{H}$ ). tively (geometric means $\pm \mathrm{SD}$ ). Response phase relative to linear acceleration averaged leads of $1.60 \pm 7.45^{\circ}$ (regular) and $28.34 \pm 16.11^{\circ}$ (irregular). Gain and phase differences between regular and irregular otolith afferents were significant (Wilcoxon's test, $p<0.001$ ). These gain and phase values are similar to those reported previously in chinchilla (Goldberg et al., 1990a), squirrel monkey (Fernández and Goldberg, 1976a-c), and rhesus monkey (Jamali et al., 2009). 
The preferred translation response directions are illustrated in Figure $2 C$. Of these, 21 had PDs within $\pm 30^{\circ}$ of the horizontal plane and were thus classified as "utricular" afferents. The remaining 28 fibers were characterized as "saccular" afferents. As illustrated in Figure 2D, which plots the mean firing rate (100 ms window) centered around the response peak minus spontaneous activity versus spontaneous activity minus the respective mean firing rate centered around the response trough, inhibitory responses (corresponding to decreases in firing rate from spontaneous activity during response trough) were lower in magnitude compared with excitatory responses (response peak) (paired $t$ test, $p<0.001$ ). Linear regression slope was 1.13 [95\% confidence interval $(\mathrm{CI})=0.94,1.35]$. Two utricular and two saccular afferents (all irregularly firing) silenced their activity during a portion of the inhibitory response cycle (Fig. $2 A, B, D$, open symbols). Mean spontaneous discharge rate for our sample of otolith afferents was $69.2 \pm 33.6$ spikes/s (Fig. 2 E), consistent with previous reports (squirrel monkey: Fernández and Goldberg, 1976a; chinchilla: Goldberg et al., 1990a; rhesus monkey: Jamali et al., 2009). There was no significant difference in gain, phase, or spontaneous activity between utricular and saccular afferents (Wilcoxon's test, $p=0.10, p=0.45, p=0.63$, respectively), also consistent with previous findings (Fernández and Goldberg, 1976a; Jamali et al., 2009).

Having established that our sample of macaque otolith afferents had all of the basic response properties similar to those reported previously in the literature, our presentation will next focus on the main goal of this study, which was to measure otolith detection thresholds.

\section{Full-cycle direction detection thresholds}

The goal of this analysis (full-cycle direction detection thresholds) was to evaluate how reliably each fiber can discriminate peak from trough activation (e.g., leftward from rightward linear acceleration). The main experimental protocol used $1 \mathrm{~Hz}$ linear acceleration oscillations at different amplitudes $(0.005-0.16 \mathrm{G})$, as illustrated for the example otolith afferent in Figure $1 B$. LR stimulation was tested in $39(79.6 \%)$ of the cells, NO stimulation was tested in $31(63.3 \%)$ of the cells, and DV direction was tested in $25(51.0 \%)$ of the cells. To quantify full-cycle detection thresholds, we first converted the firing rate into a smooth, continuous function using a Kaiser filter (Sadeghi et al., 2007), as illustrated in Figure $1 B$ (gray curves superimposed on IFR) and then applied ROC analysis to measure the sensitivity of the cell in discriminating oppositely directed linear accelerations (i.e., left/right, front/ back, and/or up/down).

We have illustrated the ROC analysis procedure using the LR direction responses of our example cell. The continuous response variable was converted into a two-alternative forced-choice (2AFC) format by measuring mean firing rate during $100 \mathrm{~ms}$ time window centered around the peak and trough of the sinusoidal modulation (Fig. 3A). This was done for each response cycle and for each stimulus magnitude. We then grouped together all firing rates within each peak (filled bars) and trough (open bars), starting with the largest stimulus magnitude $(0.16 \mathrm{G})$ and extending all the way to the smallest stimulus magnitude $(0.005 \mathrm{G})$, as illustrated in Figure $3 B$. This example cell increased its firing rate during rightward (positive) linear acceleration (Fig. 3C, black circles and line).

The purpose of the ROC analysis is to quantify the sensitivity of the neuron to small changes in the direction of linear acceleration (i.e., how reliably the neuron can discriminate activation resulting from oppositely directed linear accelerations, such as
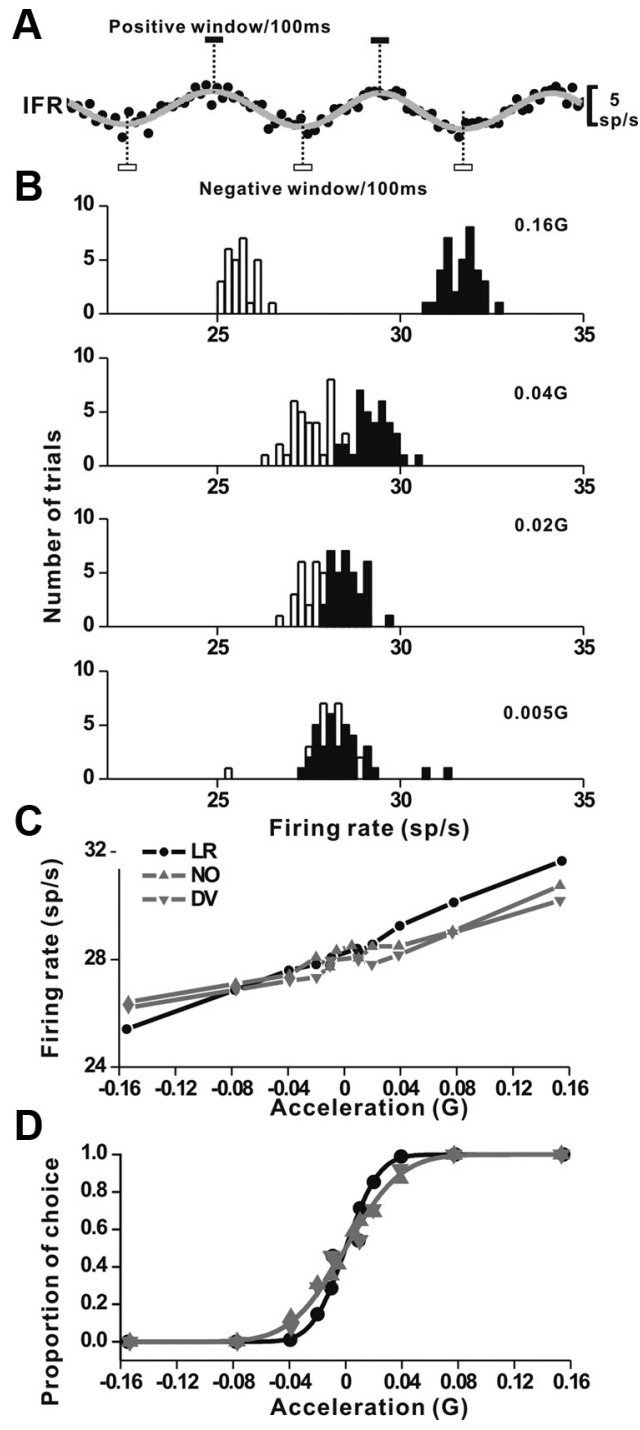

Figure 3. Quantification of full-cycle direction detection threshold for the cell in Figure 1.A, Example IFR (LR motion direction, $0.16 \mathrm{G}$ ) with superimposed gray line illustrating Kaiser filter response (see Materials and Methods). Bars mark $100 \mathrm{~ms}$ intervals used to compute mean firing rates for peak (filled bar) and trough (open bars) responses, analyzed cycle by cycle. $\boldsymbol{B}$, Firing rate distributions for four pairs of stimulus magnitudes: $0.16,0.04,0.02$, and $0.005 \mathrm{G}$. Data shown are during $L R$ motion, with filled bars corresponding to rightward (peak) and open bars corresponding to leftward (trough) responses. C, Response magnitude tuning curves for three stimulus directions: LR, NO, and DV. Positive directions are rightward, forward, and upward, respectively. $\boldsymbol{D}$, Example neurometric functions showing proportion rightward (LR, black circles), forward (NO, gray triangles), and upward (DV, gray inverted triangles) direction decisions of an ideal observer as a function of linear acceleration magnitude, computed from the magnitude tuning curve responses from $C$. Each data point corresponds to an ROC value computed from a pair of firing rate distributions like those shown in each row of $\boldsymbol{B}$. Solid lines show cumulative Gaussian fits to the neurometric functions.

acceleration from deceleration) and to measure the smallest linear acceleration magnitude for direction detection based on an ideal observer. For this purpose, one needs to quantify the overlap of two firing rate distributions, corresponding to each pair of peak/trough responses (Fig. $3 B$ ). When the stimulus magnitude is large (i.e., $0.16 \mathrm{G}$ ), the firing rate distributions do not overlap. As a result, an ideal observer would be able to distinguish whether a given firing rate came from the leftward or rightward distribution with a high degree of certainty. However, when the stimulus magnitude is small (i.e., $0.005 \mathrm{G}$ ), the firing rate distributions overlap extensively, thus making the task of an ideal observer 
difficult. Note that discriminability is determined by the separation of these two distributions, i.e., it depends on both the mean difference and the variance of the two distributions (this property is discussed further below).

This intuition is quantified using ROC analysis (Britten et al., 1992; Gu et al., 2007; Liu et al., 2010). For each pair of peak/trough response distributions, an ROC value is computed that reflects the probability that an ideal observer could accurately report whether the linear acceleration was "rightward" or "leftward" based on a draw from the firing rate distribution of the neuron. "Neurometric" functions can then be constructed by plotting ROC values for each pair of stimulus magnitudes (Fig. 3D). Quantification of this relationship captures the sensitivity of the neuron to directional signals in the same manner that psychometric functions capture perceptual sensitivity to directional signals in a 2AFC task (MacNeilage et al., 2010b). The neurometric function is summarized by fitting a cumulative Gaussian function to the data (Fig. 3D, black circles and line). Neuronal direction detection threshold is then defined as the SD of the underlying Gaussian fit (corresponding to $84 \%$ correct). Thus, for each stimulus direction tested in each cell, we obtain a neuronal threshold. This particular example cell modulated significantly (Fig. $1 A$ ), exhibited approximately linear response tuning (Fig. $3 C$ ), and had relatively small direction detection thresholds (Fig. 3D) for all three cardinal stimulus directions: $\sigma=18.64 \mathrm{~cm} / \mathrm{s}^{2}$ (LR), 30.41 $\mathrm{cm} / \mathrm{s}^{2}(\mathrm{NO})$, and $30.41 \mathrm{~cm} / \mathrm{s}^{2}$ (DV).

The direction detection thresholds for all of the macaque otolith afferents are summarized in Figure 4 (see also Table 1). When compared as two groups, irregular otolith afferents were characterized by lower thresholds than regular otolith afferents (geometric mean $\pm \mathrm{SD}, 16.05 \pm$ 2.32 vs $30.33 \pm 2.23 \mathrm{~cm} / \mathrm{s}^{2}$, respectively; Wilcoxon's test, $p<0.001$; Fig. $4 A$, marginal histograms). However, there was no significant dependence of threshold on $\mathrm{CV}^{\star}$ (Fig. $4 A$; $\mathrm{AN}$ COVA, $\left.F_{(1,93)}=0.31, p=0.58\right)$ or spontaneous activity (ANCOVA, $F_{(1,93)}=0.24, p=0.63$ ). In addition, we found no significant threshold difference for LR, NO, and DV stimulus directions (ANCOVA main effect, $F_{(2,93)}=0.13, p=0.88$; Table 1, Fig. $4 A$, different colors). Thresholds, however, did depend on the absolute value of the difference between the tested direction and the three-dimensional PD of the cell [Fig. $4 B, \Delta(3 \mathrm{D}-\mathrm{PD})$ ], such that the smallest threshold was observed when the tested direction was parallel or anti-parallel to the PD of the cell [i.e., when $\Delta(3 \mathrm{D}-$ $\mathrm{PD})=0^{\circ}$ or $180^{\circ}$. This result is not surprising because the closer to the $\mathrm{PD}$, the larger the neuronal gain, thus the smaller the neuronal threshold.
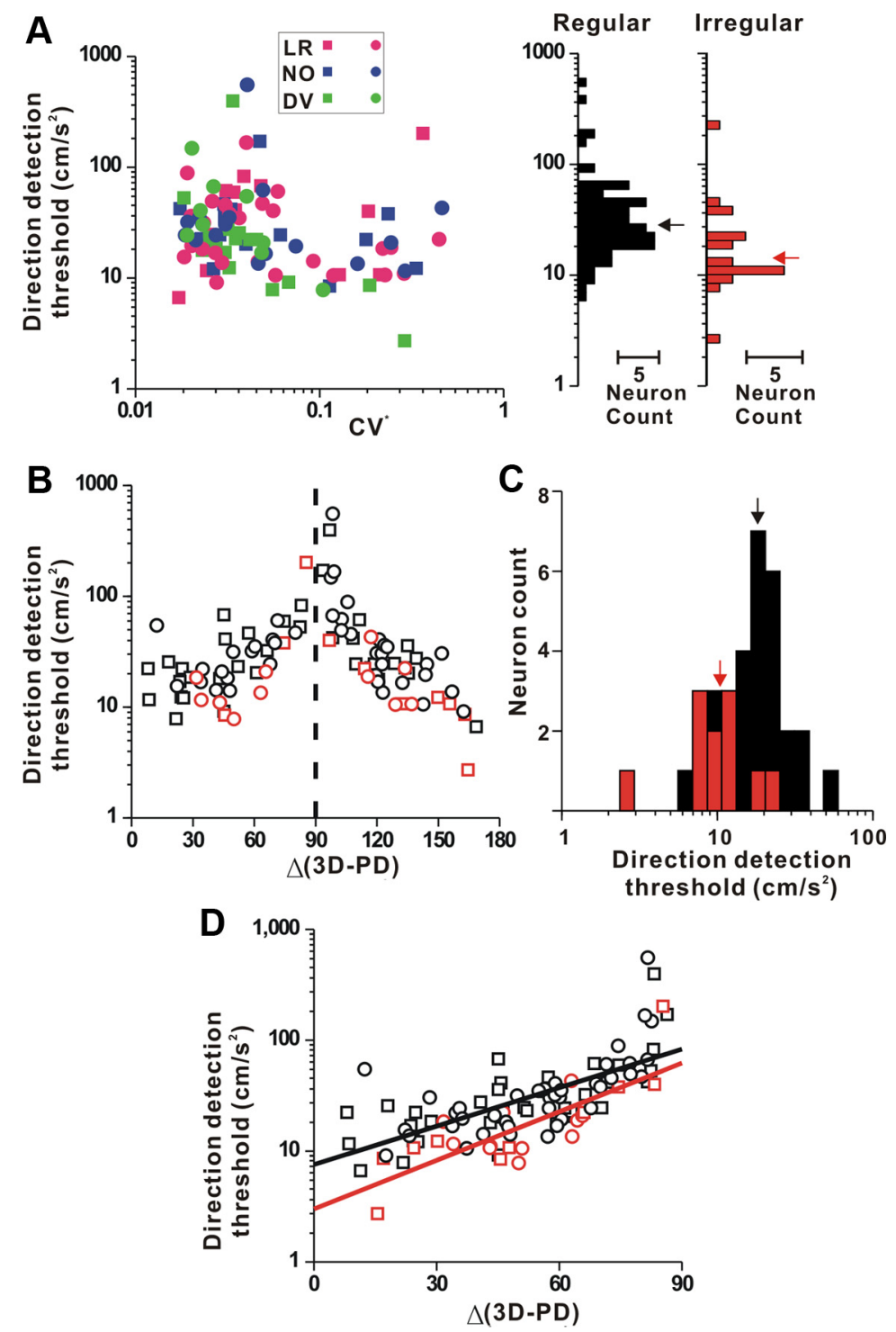

Figure 4. Summary of full-cycle direction detection thresholds $(n=49) . A$, Neuronal threshold as a function of $C V^{*}$, color coded according to stimulus direction (LR, magenta; NO, blue; DV, green). Marginal histograms on the right show threshold distributions, separately for regular (black) and irregular (red) otolith afferents (arrows illustrate geometric means). $\boldsymbol{B}$, Neuronal threshold as a function of the absolute difference between the tested direction and the three-dimensional PD of the cell, $\Delta(3 D-P D)$, with data Black symbols/bars, Regular otolith afferents $\left(\mathrm{CV}^{*}<0.1\right)$; red symbols/bars, irregular otolith afferents $\left(\mathrm{CV}^{*}>0.1\right)$. Different symbols are used for different animals (squares, monkey $\mathrm{K}$; circles, monkey $\mathrm{H}$ ).

Because neuronal threshold depends on the direction of the linear acceleration stimulus relative to the direction of maximal gain, it is important to estimate direction thresholds for stimulation along the $\mathrm{PD}$ of the cell. We approached this question in two different ways. First, Figure $4 C$ shows the distribution of thresholds along the cardinal direction that was the closest to the PD of the cell in 3D. Neuronal thresholds averaged $17.35 \pm 1.58$ and $10.13 \pm 1.67 \mathrm{~cm} / \mathrm{s}^{2}$ (geometric mean $\pm \mathrm{SD}$ ) for regular and irregular otolith afferents, respectively (Wilcoxon's test, $p=$ 0.006; Table 1). Second, we folded the data along the $90^{\circ}$ abscissa of Figure $4 B$, such that we could now fit a linear regression to the threshold dependence on $\Delta(3 \mathrm{D}-\mathrm{PD})$ (Fig. $4 D)$. The intercept of a type II regression fitted to the data gives an estimate of the 
Table 1. Summary of full-cycle direction detection thresholds (in units of $\mathrm{cm} / \mathrm{s}^{2}$ )

\begin{tabular}{|c|c|c|}
\hline & \multicolumn{2}{|l|}{ Otolith afferents } \\
\hline & Regular & Irregular \\
\hline LR & $\begin{array}{l}29.54 \pm 2.16 \text { (geometric SD) } \\
\quad(n=29)\end{array}$ & $\begin{array}{l}19.76 \pm 2.42^{a} \text { (geometric SD) } \\
\quad(n=10)\end{array}$ \\
\hline NO & $32.86 \pm 2.17(n=25)$ & $18.27 \pm 1.72(n=8)$ \\
\hline DV & $28.65 \pm 2.36(n=22)$ & $5.67 \pm 1.68(n=3)$ \\
\hline $\begin{array}{l}\text { LR/NO/DV direction with } \\
\text { largest response (Fig. 4C) }\end{array}$ & $\begin{array}{l}17.35 \pm 1.58 \text { (geometric SD) } \\
\quad(n=30)\end{array}$ & $\begin{array}{l}10.13 \pm 1.67 \text { (geometric SD) } \\
\quad(n=11)\end{array}$ \\
\hline $\begin{array}{l}\text { Three-dimensional PD } \\
\text { (intercept in Fig. 4D) }\end{array}$ & $7.54(4.70-11.16)(95 \% \mathrm{Cl})$ & $3.01(1.44-6.06)(95 \%$ Cl) \\
\hline
\end{tabular}
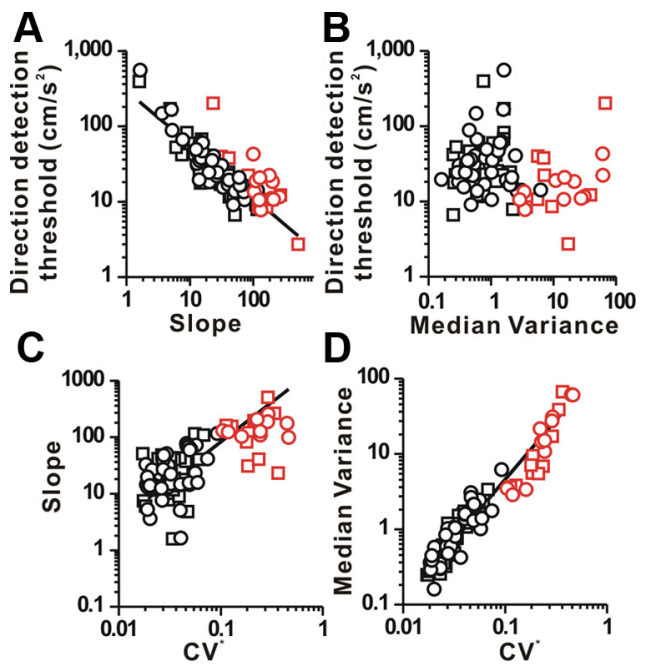

Figure 5. Parameters influencing neuronal threshold. $A, B$, Dependence of neuronal threshold on linear acceleration magnitude tuning curve slope $(\boldsymbol{A})$ and median variance $(\boldsymbol{B})$, with data from each cell shown for one to three motion directions. Median variance is computed for each direction from multiple response amplitude distributions, like those in Figure 3B. C, Correlation of magnitude tuning curve slope and $\boldsymbol{D}$, median variance with $\mathrm{CV}^{*}$. Black symbols, Regular otolith afferents $\left(\mathrm{CV}^{*}<0.1\right)$; red symbols, irregular otolith afferents $\left(\mathrm{CV}^{*}>0.1\right)$. Different symbols are used for different animals (squares, monkey K; circles, monkey H). Solid lines illustrate type Il linear regressions plotted through all data (all directions, all neurons).

population neuronal threshold for $\Delta(3 \mathrm{D}-\mathrm{PD})=0$, i.e., along the PD. These values $( \pm 95 \% \mathrm{CI})$ were $7.54(4.70-11.16)$ and 3.01 $(1.44-6.06) \mathrm{cm} / \mathrm{s}^{2}$ for regular and irregular afferents, respectively (Table 1). Note that irregular otolith afferents have consistently lower thresholds, independently of $\Delta(3 \mathrm{D}-\mathrm{PD})$.

Neuronal thresholds can depend on two properties that determine how much the two ROC distributions overlap (Fig. 3B): the difference in their means and their width (variance). The difference in the distribution means is directly related to the slope (steepness) of the magnitude tuning curve (Fig. 3C), a parameter that is related to response gain. In addition to the difference in the means, the variance of the spike count also influences the extent of overlap between the two choice distributions. In principle, higher sensitivity could arise either attributable to a sharp magnitude tuning curve (i.e., high gain) or a low response variance. Thus, to understand how neuronal sensitivity varies among cells, we have considered each of these factors separately.

Figure 5, $A$ and $B$, plots neuronal threshold as a function of slope and variance, respectively. A significant correlation exists only for slope (Fig. 5A; type II regression, $p<0.001$ ) but not for variance (Fig. $5 B$; type II regression, $p=0.10$ ). An ANCOVA applied to these data showed that this conclusion was true for both regular and irregular otolith afferents (interaction term be- tween slope/variance and fiber type, $p=0.77)$. When plotted as a function of $\mathrm{CV}^{\star}$, both slope and variance scaled with $\mathrm{CV}^{\star}$ (Fig. $5 C, D$; type II regression, $p<0.001)$. Thus, at $1 \mathrm{~Hz}$, irregular afferents have both higher slope and higher variance than regular afferents. The fact that irregular afferents have lower thresholds than regular otolith afferents suggests that, at least for $1 \mathrm{~Hz}$, the higher slope counterbalances the higher response variance. It is possible that threshold differences between regular and irregular otolith afferents depend on frequency. Specifically, gain increases with frequency are larger for irregular than regular afferents, but whether and how spike count variance varies with frequency is currently unknown. If spike count variance is independent of frequency, then it is likely that irregular afferents have lower thresholds at high frequencies and higher thresholds at low frequencies than regular otolith afferents.

\section{Half-cycle detection thresholds}

The analyses summarized above compute the sensitivity of an ideal observer to detect linear acceleration direction (i.e., left/ right, fore/aft, or up/down) based on the spike count activity of otolith afferents. In addition, it is of interest to also characterize how reliably the neuron can discriminate peak or trough activation from spontaneous activity (half-cycle detection thresholds). This comparison can be made using a similar (ROC) analysis, in which the excitatory (peak) or inhibitory (trough) response of each fiber is compared with its spontaneous activity.

Other than differences in magnitude, results from half-cycle detection threshold analyses were overall similar to those reported above for full-cycle detection thresholds (Fig. 6, Table 2). Regular afferents tended to have higher half-cycle thresholds than irregular otolith afferents (Wilcoxon's test, $p<0.001$ for excitatory detection, $p=0.07$ for inhibitory detection), although there was no significant dependence of threshold on $\mathrm{CV}^{*}$ (Fig. $6 A$, ANCOVA, $F_{(1,93)}=2.49, p=0.12$; Fig. $6 B, F_{(1,93)}=2.57, p=$ 0.11 ). In addition, we found no significant difference in half-cycle threshold for LR, NO, and DV stimulus directions (ANCOVA main effect, $F_{(2,93)}=0.04, p=0.96$; Table 2, Fig. $6 \mathrm{~A}$, different colors). Half-cycle excitatory detection thresholds did not depend on spontaneous firing rate (Spearman's rank correlation, $p=0.13$ for excitatory detection and $p=0.29$ for inhibitory detection).

The relationship between excitatory and inhibitory detection thresholds is shown in Figure 6C. On average, inhibitory halfcycle thresholds were twice as large as excitatory half-cycle thresholds (paired $t$ test, $p<0.001$ ), and the $95 \%$ CI of the linear regression slope did not include 1 (type II linear regression; $r=$ $0.61, p<0.001$, slope $=2.04,95 \% \mathrm{CI}=1.45,2.88)$. Similar to the full-cycle direction detection thresholds, half-cycle detection thresholds depended only on excitatory/inhibitory neuronal firing rate slope (type II regression, $p<0.001$ ) but not on variance (type II regression, $p>0.25$ ). An ANCOVA applied to these data showed that this conclusion was true for both regular and irregular afferents (interaction terms between slope/variance and fiber type, $p=0.73$ ). Half-cycle excitatory detection thresholds along the cardinal direction that was the closest to the $\mathrm{PD}$ of the cell in $3 \mathrm{D}$ averaged $28.42 \pm 1.56$ and $17.43 \pm 1.89 \mathrm{~cm} / \mathrm{s}^{2}$ (geometric mean $\pm \mathrm{SD}$ ), for regular and irregular otolith afferents, respectively (Wilcoxon's test, $p=0.04$ ). Inhibitory half-cycle detection thresholds along the cardinal direction with the largest gain averaged $40.37 \pm 2.21$ and $27.87 \pm 2.32 \mathrm{~cm} / \mathrm{s}^{2}$ (Table 2).

Similar to the full-cycle thresholds, half-cycle detection thresholds depended on the difference between the tested direction and the three-dimensional PD of the cell (Fig. 6D), such that 
the smallest threshold was observed when the tested direction was parallel or anti-parallel to the PD of the cell. The intercepts of a type II linear regression on folded $\Delta(3 \mathrm{D}-\mathrm{PD})$ half-cycle excitatory detection thresholds are 16.77 (12.01-22.13, 95\% CI) and 5.94 (2.34$16.42,95 \% \mathrm{CI}) \mathrm{cm} / \mathrm{s}^{2}$ for regular and irregular afferents, respectively (Table 2). Note that excitatory/inhibitory thresholds were not significantly different when extrapolated along the PD (overlapping 95\% CIs; Table 2).

\section{Nonlinearities in magnitude tuning curves}

The large inhibitory half-cycle thresholds (some $\gg 500 \mathrm{~cm} / \mathrm{s}^{2}$; Fig. $6 \mathrm{~B}$ ) prompted us to further investigate potential nonlinearities, already suggested by Figure $2 D$. Amplitude nonlinearities were reported previously for large linear accelerations (Fernández et al., 1972; Fernández and Goldberg 1976a,b). Representative magnitude tuning curves from six otolith afferents showing evidence for nonlinear magnitude tuning curves are shown in Figure $7 A-F$. Some otolith afferents exhibited clear inhibitory response saturation, even for the low linear acceleration amplitudes used here. To quantify these nonlinearities, we fitted both linear and quadratic equations to these firing rate versus linear acceleration amplitude relationships.

Figure $8 \mathrm{~A}$ summarizes in a scatter plot format the relationship between the correlation coefficients from the linear $\left(R^{2} \_\mathrm{L}\right)$ and quadratic $\left(R^{2} \_\mathrm{Q}\right)$ model fits. The quadratic model fit was significantly better (based on AIC; see Materials and Methods) than the linear model fit in 78 of 96 tuning curves (Fig. $8 \mathrm{~A}$, filled symbols), whereas the linear model provided as good a fit for only 18 of 96 (19\%) tuning curves (Fig. $8 \mathrm{~A}$, open symbols). With the exception of a few tuning curves along directions with very small gains $(<10$ spikes/s/G), the quadratic model fit gave $R^{2} \_\mathrm{Q}>0.7$ (Fig. $8 B$ ). The improvement in correlation coefficient with the quadratic model $\left(R^{2}{ }_{-} \mathrm{Q}-R^{2} \_\mathrm{L}\right)$ did not depend on $\mathrm{CV}^{\star}(r=0.09, p=0.4$, type II regression) but was larger for directions with lower gain than directions with higher gain (Fig. 8C, Dir3 vs Dir1, respectively). Indeed, the mean $R^{2}{ }_{-} \mathrm{L}$ for cardinal directions with the largest response gain was $R^{2}{ }_{-} \mathrm{L}=0.96 \pm 0.008$ (SE) (range, $0.78-$ 1). Nevertheless, as illustrated in Figure $8 D$, the ratio of excitatory half-cycle to inhibitory half-cycle thresholds is significantly correlated with the improvement in correlation coefficient, $R^{2} \mathrm{Q}_{-}$ $R^{2} \_$L $(r=-0.44, p<0.001$, type II regression; directions with gain $<10$ spikes/s/G were excluded). That is, the larger the inhibitory response saturation, the larger the difference between the two half-cycle thresholds.
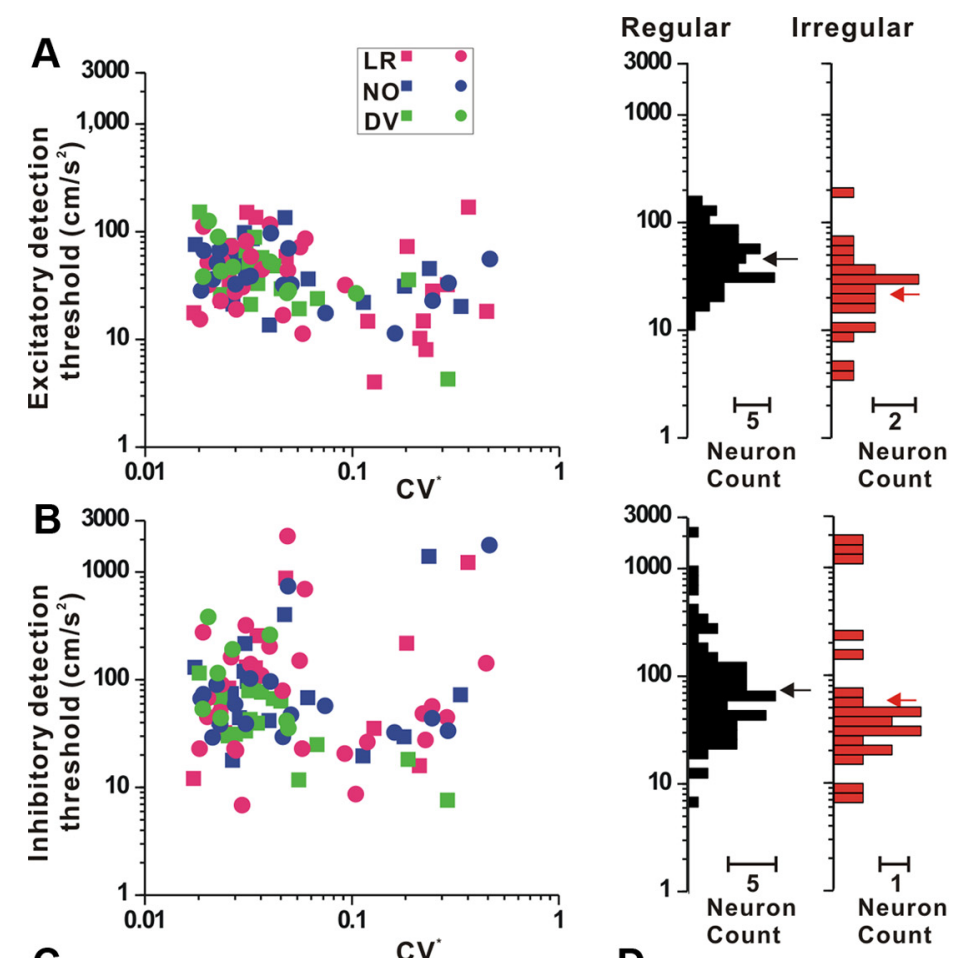
responses as a function of $\mathrm{CV}^{*}$, color coded according to stimulus direction ( $\mathrm{LR}$, magenta; N0, blue; DV, green). Marginal histograms on the right show threshold distributions, separately for regular (black) and irregular (red) otolith afferents (arrows illustrate slope. Solid line plots type II linear regression. $D$, Excitatory detection threshold as a function of the absolute difference between the

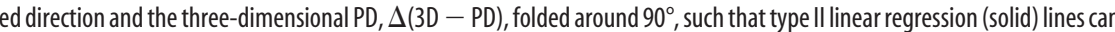
egular otolith afferents $\left(\mathrm{CV}^{*}<0.1\right)$; red symbols/bars, irregular otolith afferents $\left(\mathrm{CV}^{*}>0.1\right)$. Different symbols are used for different animals (squares, monkey $\mathrm{K}$; circles, monkey $\mathrm{H}$ ).

\section{Dependence on analysis parameters}

As important as it might be to correlate neuronal firing rate properties with perceptual measures, caution is called for when neural data are not simultaneously collected along with behavioral data (Gu et al., 2007, 2008). This is particularly problematic because (1) it is unclear which firing rate property is decoded by downstream neurons and perceptual neural correlates and (2) there are multiple ways of quantifying firing rates. To illustrate the latter point, we have plotted geometric mean \pm SD of population fullcycle direction detection thresholds (filled symbols/solid lines indicate regular otolith afferents; open symbols/dashed lines indicate irregular otolith afferents) as a function of analysis window size (i.e., the temporal window for computing peak, through, and spontaneous firing rates) for different filtering methods used to analyze neural responses (Fig. 9). Although the window size for 
Table 2. Summary of half-cycle detection thresholds (in units of $\mathrm{cm} / \mathrm{s}^{2}$ )

\begin{tabular}{|c|c|c|c|c|}
\hline & \multicolumn{4}{|l|}{ Otolith afferents } \\
\hline & \multicolumn{2}{|l|}{ Regular } & \multicolumn{2}{|l|}{ Irregular } \\
\hline & Excitatory & Inhibitory & Excitatory & Inhibitory \\
\hline LR & $42.15 \pm 2.00(n=29)$ & $88.56 \pm 3.69(n=29)$ & $20.79 \pm 2.80(n=10)$ & $67.38 \pm 3.38(n=10)$ \\
\hline NO & $46.70 \pm 1.73(n=25)$ & $74.87 \pm 2.23(n=25)$ & $27.38 \pm 1.60(n=8)$ & $91.97 \pm 5.36(n=8)$ \\
\hline DV & $45.29 \pm 1.75(n=22)$ & $61.81 \pm 2.20(n=22)$ & $16.04 \pm 2.56(n=3)$ & $10.64 \pm 1.47(n=3)$ \\
\hline LR/NO/DV direction with largest response (Fig. 4C) & $28.42 \pm 1.56(n=30)$ & $40.37 \pm 2.21(n=30)$ & $17.43 \pm 1.89(n=11)$ & $27.87 \pm 2.32(n=11)$ \\
\hline Three-dimensional PD (intercept in Fig. 6D) & 16.77 (12.01-22.13) (95\% Cl) & $16.15(8.71-27.72)(95 \% \mathrm{Cl})$ & $5.94(2.34-16.42)(95 \% \mathrm{Cl})$ & $4.66(1.71-13.52)(95 \% \mathrm{Cl}$ \\
\hline
\end{tabular}

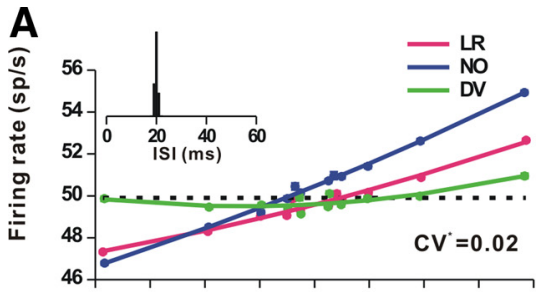

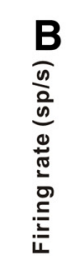
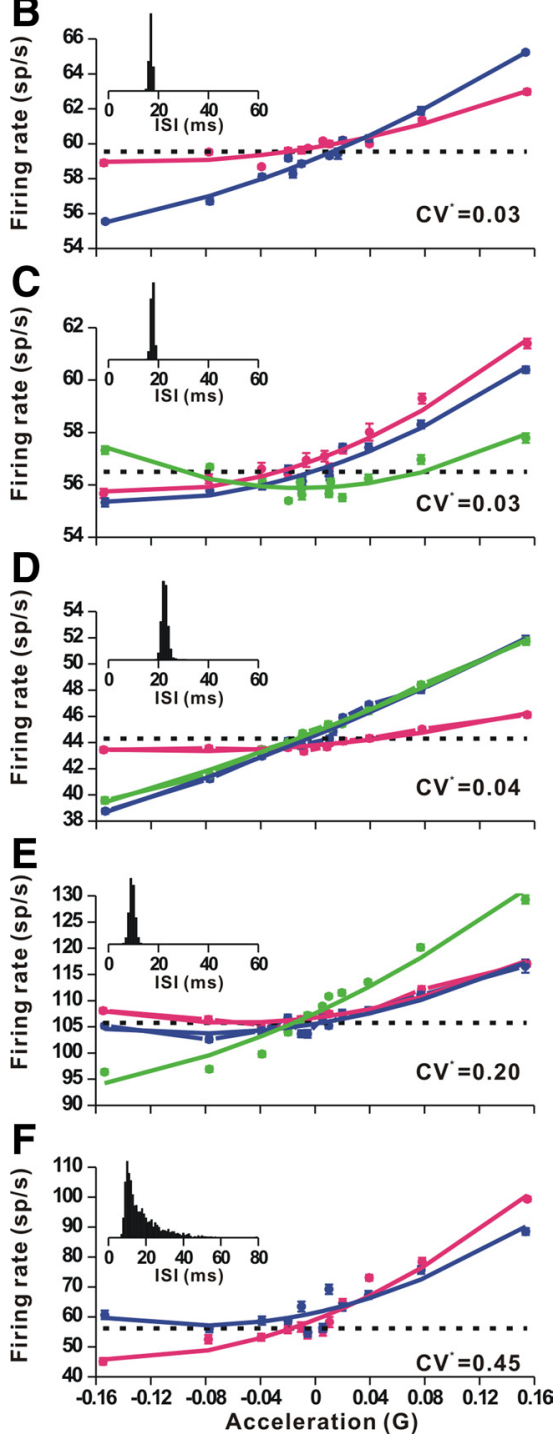

Figure 7. $A-\boldsymbol{F}$, Example magnitude tuning curves for six representative otolith afferents with significant nonlinearities, plotted for LR, NO, and DV directions (positive values: rightward, forward, and upward stimuli; negative values: leftward, backward, and downward stimuli). Solid lines illustrate quadratic fit. Horizontal dashed lines illustrate spontaneous (baseline) activity. Insets illustrate baseline ISI distribution.
A

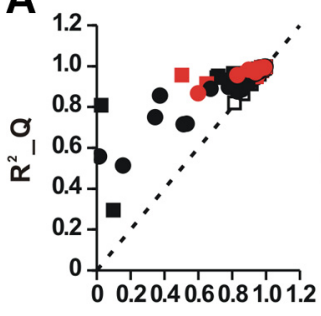

B
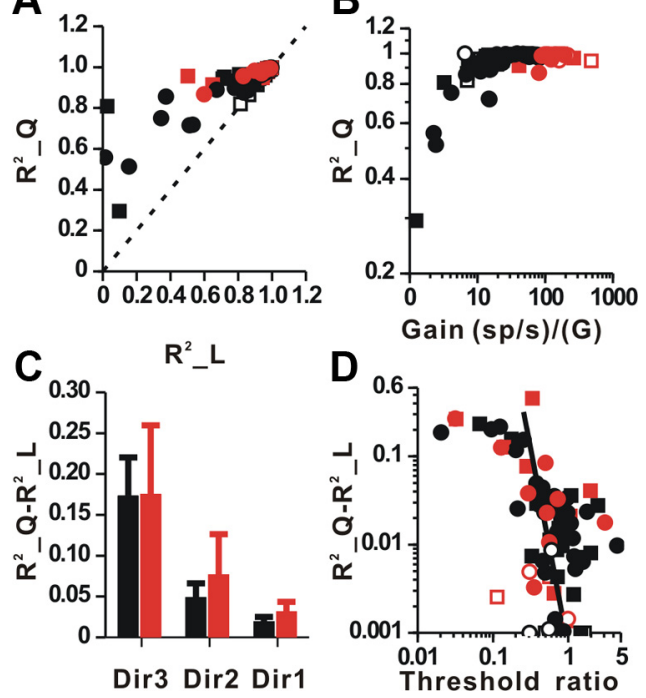

D

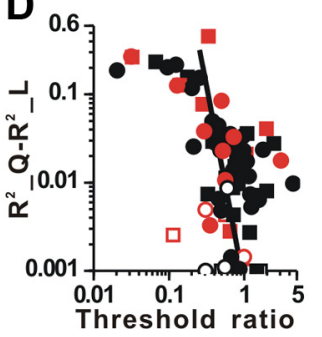

Figure 8. Summary of nonlinearities in magnitude tuning curves. $A$, Scatter plot of quadratic and linear correlation coefficients $\left(R^{2} \_\right.$and $R^{2} L$, respectively) fitted to magnitude tuning curves of all cardinal directions tested (unity dotted line is also shown). $\boldsymbol{B}$, Scatter plot of quadratic correlation coefficient $\left(R^{2} \_\right.$) versus response gain along the respective direction. $C$, Bar graph of mean $\pm S D$ difference between the two correlation coefficients $\left(R^{2} \_-R^{2} \_\right.$) grouped according to maximum (Dir1) and minimum (Dir 3) cardinal response direction, summarized separately for regular (black bars) and irregular (red bars) afferents. $D$, Scatter plot of the difference between the two correlation coefficients $\left(R^{2}{ }_{-} Q-R^{2} L\right.$ ) versus the ratio of excitatory half-cycle to inhibitory half-cycle threshold (data from directions with gain $<10$ spikes $/ \mathrm{s} / \mathrm{G}$ have been excluded). Solid line illustrates type II linear regression. For illustrative purposes, correlation coefficient differences $<0.001$ have been set at $R^{2}{ }_{-} Q-R^{2}{ }_{-} L=0.001$. Black symbols/bars, Regular otolith afferents $\left(\mathrm{CV}^{*}<0.1\right)$; red symbols/bars, irregular otolith afferents $\left(\mathrm{CV}^{*}>0.1\right)$. Different symbols are used for different animals (squares, monkey $\mathrm{K}$; circles, monkey $\mathrm{H})$.

computing mean firing rates for ROC distribution comparisons has overall a very small effect on neuronal threshold, the filtering parameters used to compute spike count are critical to determining the actual values of the computed neuronal threshold. In the presentation here, we have used the Kaiser filter for two reasons: (1) because it has been previously used by the only other study computing peripheral vestibular thresholds (for semicircular canal afferents; Sadeghi et al., 2007), and (2) because it gives the smallest neuronal threshold (Fig. 9, black solid line), likely because of reducing the variance attributable to stronger filtering.

At present, we are agnostic about the neuronal parameters used by the brain to extract behavioral measures. Thus, it is not our goal to make an intelligent choice about the different analysis options. However, the variability shown in Figure 9 illustrates that it will be unfair to directly and quantitatively compare the absolute numbers of neuronal thresholds with various psychophysical measures. Although extreme caution is advised for making direct comparisons, the present findings are the first to 


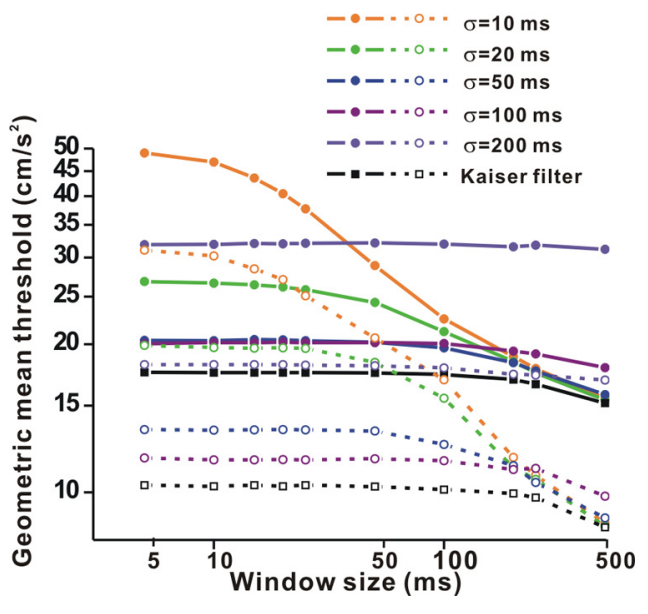

Figure 9. Dependence of population (geometric mean) full-cycle detection threshold (along the cardinal direction with the largest response) on analysis parameters, i.e., window size for computing mean firing rate (bars in Fig. $3 A$ ) and filtering method (Kaiser filter and spike density functions with different widths, $\sigma$; see Materials and Methods). Data are shown separately for regular (filled symbols, solid lines) and irregular (open symbols, dashed lines) otolith afferents. The analyses in previous figures used the Kaiser filter and a window size of $100 \mathrm{~ms}$.

quantitatively characterize both spike count variability and neuronal sensitivity around psychophysical threshold in the peripheral otolith system. These data will form the basis for comparing neuronal discriminability in central vestibular neurons. Notably, the reported lower thresholds for irregular compared with regular otolith afferents persist and remain a consistent finding, regardless of the method used to measure mean firing rate.

\section{Discussion}

We have measured linear acceleration detection thresholds using ROC analysis to quantify how reliably macaque primary otolith afferents can discriminate activation resulting from oppositely directed linear accelerations (e.g., acceleration from deceleration). Such full-cycle direction detection thresholds averaged $17.35 \pm 1.58 \mathrm{~cm} / \mathrm{s}^{2}(0.0177 \mathrm{G}$, regular otolith afferents $)$ and $10.13 \pm 1.67 \mathrm{~cm} / \mathrm{s}^{2}(0.0103 \mathrm{G}$, irregular otolith afferents) along the cardinal axis with maximum response. When extrapolated along the PD, full-cycle detection thresholds were $7.54 \mathrm{~cm} / \mathrm{s}^{2}$ $\left(0.0077 \mathrm{G}\right.$, regular afferents) and $3.01 \mathrm{~cm} / \mathrm{s}^{2}(0.0031 \mathrm{G}$, irregular afferents). The fact that irregular otolith afferents have lower thresholds than regular afferents might appear surprising because of their higher response variability (Fig. 5D). Lower thresholds are observed because irregular afferents have sufficiently higher gains at $1 \mathrm{~Hz}$, a fact that outweighs the higher variance in their contribution to neuronal sensitivity. We have also measured half-cycle detection thresholds by comparing peak and trough responses with spontaneous activity. Theoretically, if (1) mean peak and trough responses are symmetrically spaced around spontaneous activity and (2) the spike count variability of peak, trough, and spontaneous activity are all equal, then half-cycle detection thresholds should be twice as large as full-cycle detection thresholds (because firing rate distribution means would differ by half as much as those for full-cycle detection thresholds). Indeed, half-cycle detection thresholds were approximately double (Table 2), although inhibitory half-cycle thresholds were twice as large as excitatory half-cycle thresholds because of amplitude nonlinearities.

Importantly, we also show that the actual values of neuronal threshold might be of lesser importance than the general patterns of responsiveness and comparisons between groups of afferents (i.e., regular/irregular, direction of motion). This is because it is unknown what parameter of neural firing is used and decoded by the brain to detect or discriminate motion. This has been illustrated by varying the analysis window size and the firing rate filter and comparing population thresholds as a function of these parameters (Fig. 9). While keeping these caveats in mind, we next compare the present findings with perceptual thresholds of linear acceleration from human psychophysical studies.

\section{Perceptual detection thresholds of linear acceleration}

The initial studies characterizing perceptual detection thresholds during linear motion resulted in a wide range of reported values $\left(1.4-18 \mathrm{~cm} / \mathrm{s}^{2}\right)$. This diversity in reported thresholds was primarily attributable to the use of multiple psychophysical methods and equipment (Walsh, 1961, 1962; Young and Meiry, 1968; Greven et al., 1974; Jones and Young, 1978) (for review, see Guedry 1974). Here we will focus our discussion on four studies that have used similar stimuli (a single discrete movement having a bell-shaped linear velocity profile and biphasic acceleration trajectory that approximates a single sine wave) and quantitative methods based on signal detection theory (Benson et al., 1986; Zupan and Merfeld, 2008; MacNeilage et al., 2010a,b).

Benson et al. (1986) used 3 s discrete displacements to discriminate the direction of LR, NO, and DV movements. They reported $67 \%$ correct thresholds of $0.006 \mathrm{G}$ for discriminating movement along the LR and NO axes in upright subjects and $0.015 \mathrm{G}$ for DV axis movement in supine (back-down) subjects. The authors also reported sharp decreases of behavioral threshold as a monotonic function of stimulus duration during bellshaped velocity profiles ranging in duration from 1 to $7 \mathrm{~s}$ (Guedry, 1974; Benson et al., 1986) (i.e., higher frequencies of motion). More recently, using a $4 \mathrm{~s}$ biphasic acceleration stimulus and a one-interval left/right discrimination task, Zupan and Merfeld (2008) reported slightly lower linear acceleration thresholds $(0.005 \mathrm{G})$ along the LR axis.

Later, MacNeilage et al. (2010b) also measured direction discrimination thresholds during LR and DV movements using a task and analysis similar to the one used by Benson et al. (1986). Subjects experienced a single $1 \mathrm{~s}$ movement and indicated whether the movement was in the positive or negative direction along that axis (i.e., leftward or rightward and upward or downward), whereas displacement (and thus peak acceleration) was varied from trial to trial according to a staircase procedure. The percentage of responses for one alternative was plotted as a function of the linear acceleration amplitude, and threshold was defined as the $\operatorname{SD}(\sigma)$ of the best-fitting cumulative Gaussian (corresponding to $84 \%$ correct, similar to the present analysis). Human direction discrimination thresholds averaged $6.3 \pm 2.5$ $\mathrm{cm} / \mathrm{s}^{2}(0.006 \mathrm{G})$ for inertial motion along the LR axis and $9.7 \pm$ $3.4 \mathrm{~cm} / \mathrm{s}^{2}(0.01 \mathrm{G})$ for inertial motion along the DV axis (with the subject sitting upright), and this difference was statistically significant (MacNeilage et al., 2010b). The DV threshold was even higher in ear-down orientations, in agreement with the findings of Benson et al. (1986). There was no difference between LR and DV directions for amplitude discrimination thresholds around a pedestal of $0.03 \mathrm{G}$ (MacNeilage et al., 2010b).

MacNeilage et al. (2010a) also quantified minimum linear acceleration for absolute detection in a set of experiments characterizing canal/otolith interactions in the earth-horizontal plane during $2 \mathrm{~s}$ curved path motion. Perceptual detection thresholds $\left(d^{\prime}=1\right)$ averaged $7.07 \pm 5.05 \mathrm{~cm} / \mathrm{s}^{2}(0.007 \mathrm{G})$ for translation along the NO axis (MacNeilage et al., 2010a). Because all trials included actual platform motion, these measurements avoid the 
confound of background vibration and other extraneous cues that otherwise challenge quantification of vestibular detection thresholds. Both MacNeilage et al. (2010a,b) studies used a motion platform identical to the one used here, although the motion profile was a transient bell-shaped linear velocity (1 or $2 \mathrm{~s}$ in duration). In addition, note that, in the study by MacNeilage et al. (2010a), subjects were presented with both peak and trough (biphasic linear acceleration stimulus), and they could perform the task by detecting either one.

Direct comparisons between these psychophysical studies and the present findings are not possible because of at least three reasons: (1) neuronal threshold values depend on analysis parameters (Fig. 9); (2) there might be potential differences between humans and monkeys; and (3) there are fundamental differences in stimulus design. In particular, the coarse direction discrimination threshold experiments discussed above (Benson et al., 1986; Zupan and Merfeld, 2008; MacNeilage et al., 2010b) used transient linear displacements with biphasic linear acceleration profiles to discriminate the direction of the linear displacement (or linear velocity). Although these stimuli and task design parallel the one used in our neurometric analysis (e.g., the ideal observer must discriminate peak from trough activation, i.e., acceleration vs deceleration), in the psychophysical experiments, the order of acceleration/deceleration had to be identified also to specify the direction of the linear displacement. In contrast, here we have measured only the sensitivity to discriminating the direction of linear acceleration and not the order of acceleration/ deceleration. In fact, individual otolith afferents do not carry information about the order of acceleration/deceleration, a critical property that arises only centrally in vestibular cells in which neuronal activity carries linear velocity signals (Fetsch et al., 2010; Chen et al., 2011a).

Differences between detection thresholds along different axes Unlike utricular receptors, the saccular system is biased at rest because saccular afferents are stimulated by the constant force of gravity. Thus, saccular afferents will modulate around the $1 \mathrm{G}$ force of gravity. Higher gains for otolith afferents preferring horizontal versus vertical axes have been reported previously in the squirrel monkey (Fernández et al., 1972; Fernández and Golberg, 1976a), although no such difference has been observed in the rhesus monkey (present study; Jamali et al., 2009). Perhaps surprisingly, we found that not only gain and phase but also response thresholds of cells preferring vertical directions (presumably saccular afferents) were comparable with those preferring horizontal directions (presumably utricular afferents).

These findings are unexpected compared with what has been known previously from human psychophysics. As summarized above, coarse direction discrimination thresholds have been consistently reported to be lower for horizontal than vertical linear accelerations. In particular, Benson et al. (1986) reported thresholds of $0.006 \mathrm{G}$ for lateral and fore/aft movements but significantly larger thresholds $(0.015 \mathrm{G})$ for vertical translations (Benson et al., 1986). One of the reasons for this large difference was that DV thresholds were tested in supine (back-down) position, and we now know that tilted subjects have overall higher thresholds (MacNeilage et al., 2010b). However, significant differences were also reported by MacNeilage et al. (2010b) using a similar LR/DV task with subjects upright (0.006 vs $0.097 \mathrm{G}$ ).

The present findings demonstrate that the higher vertical linear acceleration direction discrimination thresholds in perception do not represent a property of the vestibular periphery. It is thus possible that these behavioral differences are attributable to either central processing or cognitive factors. Future experiments must test linear acceleration detection thresholds in other areas, most importantly vestibular-responding areas in the cortex, such as parieto-insular vestibular cortex (Guldin and Grüsser, 1998; Chen et al., 2010; Liu et al., 2011), medial superior temporal area (Gu et al., 2006, 2007; Fetsch et al., 2010; Chen et al., 2011a), and ventral intraparietal area (Schlack et al., 2002; Chen et al., 2011a,b).

\section{References}

Akaike H (1992) Data analysis by statistical models (in Japanese). No To Hattatsu 24:127-133.

Anderson JH, Blanks RH, Precht W (1978) Response characteristics of semicircular canal and otolith systems in cat. I. Dynamic responses of primary vestibular fibers. Exp Brain Res 32:491-507.

Angelaki DE, Dickman JD (2000) Spatiotemporal processing of linear acceleration: primary afferent and central vestibular neuron responses. J Neurophysiol 84:2113-2132.

Angelaki DE, Shaikh AG, Green AM, Dickman JD (2004) Neurons compute internal models of the physical laws of motion. Nature 430:560-564.

Benson AJ, Spencer MB, Stott JR (1986) Thresholds for the detection of the direction of whole-body, linear movement in the horizontal plane. Aviat Space Environ Med 57:1088-1096.

Britten KH, Shadlen MN, Newsome WT, Movshon JA (1992) The analysis of visual motion: a comparison of neuronal and psychophysical performance. J Neurosci 12:4745-4765.

Chen A, DeAngelis GC, Angelaki DE (2010) Macaque parieto-insular vestibular cortex: responses to self-motion and optic flow. J Neurosci 30:3022-3042.

Chen A, DeAngelis GC, Angelaki DE (2011a) A comparison of vestibular spatiotemporal tuning in macaque parietoinsular vestibular cortex, ventral intraparietal area, and medial superior temporal area. J Neurosci 31:3082-3094.

Chen A, DeAngelis GC, Angelaki DE (2011b) Representation of vestibular and visual cues to self-motion in ventral intraparietal cortex. J Neurosci 31:12036-12052.

De Vrijer M, Medendorp WP, Van Gisbergen JA (2008) Shared computational mechanism for tilt compensation accounts for biased verticality percepts in motion and pattern vision. J Neurophysiol 99:915-930.

Dickman JD, Angelaki DE, Correia MJ (1991) Response properties of gerbil otolith afferents to small angle pitch and roll tilts. Brain Res 556:303-310.

Fernández C, Goldberg JM (1976a) Physiology of peripheral neurons innervating otolith organs of the squirrel monkey. I. Response to static tilts and to long-duration centrifugal force. J Neurophysiol 39:970-984.

Fernández C, Goldberg JM (1976b) Physiology of peripheral neurons innervating otolith organs of the squirrel monkey. II. Directional selectivity and force-response relations. J Neurophysiol 39:985-995.

Fernández C, Goldberg JM (1976c) Physiology of peripheral neurons innervating otolith organs of the squirrel monkey. III. Response dynamics. J Neurophysiol 39:996-1008.

Fernández C, Goldberg JM, Abend WK (1972) Response to static tilts of peripheral neurons innervating otolith organs of the squirrel monkey. J Neurophysiol 35:978-987.

Fetsch CR, Rajguru SM, Karunaratne A, Gu Y, Angelaki DE, Deangelis GC (2010) Spatiotemporal properties of vestibular responses in area MSTd. J Neurophysiol 104:1506-1522.

Goldberg JM, Desmadryl G, Baird RA, Fernández C (1990a) The vestibular nerve of the chinchilla. IV. Discharge properties of utricular afferents. J Neurophysiol 63:781-790.

Goldberg JM, Desmadryl G, Baird RA, Fernández C (1990b) The vestibular nerve of the chinchilla. V. Relation between afferent discharge properties and peripheral innervation patterns in the utricular macula. J Neurophysiol 63:791-804.

Green AM, Swets JA (1966) Signal detection theory and psychophysics. New York: Wiley.

Greven AJ, Oosterveld WJ, Rademakers WJ (1974) Linear acceleration perception: threshold determinations with the use of a parallel swing. Arch Otolaryngol 100:453-459.

Gu Y, Watkins PV, Angelaki DE, DeAngelis GC (2006) Visual and nonvisual contributions to three-dimensional heading selectivity in the medial superior temporal area. J Neurosci 26:73-85.

Gu Y, DeAngelis GC, Angelaki DE (2007) A functional link between area 
MSTd and heading perception based on vestibular signals. Nat Neurosci 10:1038-1047.

Gu Y, Angelaki DE, Deangelis GC (2008) Neural correlates of multisensory cue integration in macaque MSTd. Nat Neurosci 11:1201-1210.

Guedry FE (1974) Psychophysics of vestibular sensation. In: Handbook of sensory physiology. Vestibular system, Vol 6 (Kornhuber HH, ed), pp 3-154. New York: Springer.

Guldin WO, Grüsser OJ (1998) Is there a vestibular cortex? Trends Neurosci $21: 254-259$.

Haque A, Angelaki DE, Dickman JD (2004) Spatial tuning and dynamics of vestibular semicircular canal afferents in rhesus monkeys. Exp Brain Res 155:81-90.

Jamali M, Sadeghi SG, Cullen KE (2009) Response of vestibular nerve afferents innervating utricle and saccule during passive and active translations. J Neurophysiol 101:141-149.

Jones GM, Young LR (1978) Subjective detection of vertical acceleration: a velocity-dependent response? Acta Otolaryngol 85:45-53.

Liu S, Yakusheva T, Deangelis GC, Angelaki DE (2010) Direction discrimination thresholds of vestibular and cerebellar nuclei neurons. J Neurosci 30:439-448.

Liu S, Dickman JD, Angelaki DE (2011) Response dynamics and tilt versus translation discrimination in parietoinsular vestibular cortex. Cereb Cortex 21:563-573.

Loe PR, Tomko DL, Werner G (1973) The neural signal of angular head position in primary afferent vestibular nerve axons. J Physiol 230:29-50.

MacNeilage PR, Ganesan N, Angelaki DE (2008) Computational approaches to spatial orientation: from transfer functions to dynamic Bayesian inference. J Neurophysiol 100:2981-2996.

MacNeilage PR, Turner AH, Angelaki DE (2010a) Canal-otolith interactions and detection thresholds of linear and angular components during curved-path self-motion. J Neurophysiol 104:765-773.

MacNeilage PR, Banks MS, DeAngelis GC, Angelaki DE (2010b) Vestibular heading discrimination and sensitivity to linear acceleration in head and world coordinates. J Neurosci 30:9084-9094.
Meng H, Green AM, Dickman JD, Angelaki DE (2005) Pursuit-vestibular interactions in brain stem neurons during rotation and translation. J Neurophysiol 93:3418-3433.

Paulin MG, Hoffman LF (1999) Modelling the firing pattern of bullfrog vestibular neurons responding to naturalistic stimuli. Neurocomputing 26-27:223-228.

Paulin MG, Hoffman LF (2001) State-space receptive fields of semicircular canal afferent neurons in the bullfrog. Neurocomputing 41-40:293-298.

Purcell IM, Newlands SD, Perachio AA (2003) Responses of gerbil utricular afferents to translational motion. Exp Brain Res 152:317-322.

Sadeghi SG, Chacron MJ, Taylor MC, Cullen KE (2007) Neural variability, detection thresholds, and information transmission in the vestibular system. J Neurosci 27:771-781.

Schlack A, Hoffmann KP, Bremmer F (2002) Interaction of linear vestibular and visual stimulation in the macaque ventral intraparietal area (VIP). Eur J Neurosci 16:1877-1886.

Si X, Angelaki DE, Dickman JD (1997) Response properties of pigeon otolith afferents to linear acceleration. Exp Brain Res 117:242-250.

Walsh EG (1961) Role of the vestibular apparatus in the perception of motion on a parallel swing. J Physiol 155:506-513.

Walsh EG (1962) The perception of rhythmically repeated linear motion in the horizontal plane. Br J Psychol 53:439-445.

Yakusheva T, Blazquez PM, Angelaki DE (2008) Frequency-selective coding of translation and tilt in macaque cerebellar nodulus and uvula. J Neurosci 28:9997-10009.

Young LR, Meiry JL (1968) A revised dynamic otolith model. Aerosp Med 39:606-608.

Yu XJ, Dickman JD, Angelaki DE (2011) Direction detection thresholds of macaque otolith and semicircular canal afferents. Soc Neurosci Abstr 37:579.10.

Zupan LH, Merfeld DM (2008) Interaural self-motion linear velocity thresholds are shifted by roll vection. Exp Brain Res 191:505-511. 